\title{
CONVULSIÓN FEBRIL SIMPLE
}

\author{
Andrés Quintana MD*, Marisela Gil MD**
}

\section{Resumen}

La crisis febril simple está relacionada con una gran variedad de patologías que cursan con fiebre. Su manejo amerita un enfoque claro para prevenir complicaciones, evitar el riesgo de recurrencias, optimizar los recursos y lograr una adecuada orientación de los padres. Se revisa la literatura sobre convulsión febril simple, debido a que pese a la exacta definición de ésta, sus criterios diagnósticos y tratamiento en el servicio de urgencias aun dan lugar a controversias. Es necesario unificar conceptos y actualizarlos, para disminuir el diagnóstico equivocado que conlleva a errores terapéuticos.

Palabras clave: crisis febril simple, edad, riesgo, diagnóstico, tratamiento.

Abreviaturas: CF (S), convulsión(es) febril(es) (simples).

\section{SIMPLE FEBRILE CONVULSION}

\section{Abstract}

A simple febrile crisis is related with a great variety of febrile illnesses. A clear approach to management is essential in order to prevent complications and recurrence risk, optimizing resources and achieving adequate counseling to parents. The literature on simple febrile convulsion was reviewed. Despite it has an exact definition, there are still controversies regarding its diagnostic criteria and management at the emergency ward. Unifying and updating concepts is needed to reduce wrong diagnosis leading to therapeutic errors.

Key words: simple febrile crisis, age, risk, diagnostic, treatment

Fecha recibido: noviembre 15 de 2013 - Fecha aceptado: junio 26 de 2014

* Neuropediatra Instructor Asociado, Docente de la Universidad del Quindio, Hospital San Juan de Dios, Armenia, Colombia.
** Residente III año de Pediatría. Hospital de San José. Fundación Universitaria Ciencias de la Salud. Bogotá DC. Colombia. 


\section{Introducción}

La CFS presenta una alta incidencia en la infancia (2 a 5\%) y se relaciona con una gran variedad de patologías que cursan con fiebre. ${ }^{1}$ Su manejo amerita un enfoque claro para prevenir complicaciones, evitar el riesgo de recurrencias, optimizar los recursos y lograr una adecuada orientación de los padres. A menudo estos niños ingresan y se someten a variados procedimientos diagnósticos y terapéuticos, a pesar de que no existan pruebas firmes que los sustenten. El análisis del adecuado tratamiento del niño con CF de acuerdo con la evidencia científica constituye un buen entorno para ilustrar los problemas comunes y específicos que entraña la realización de una práctica clínica basada en las mejores pruebas científicas. ${ }^{2}$

Para conceptualizar los aspectos que rodean la $\mathrm{CF}$, se realizó una búsqueda basada en Medical Subject Headings $(\mathrm{MeSH})$, después se revisaron las bases de datos en inglés de PUBMED, HINARI, OVID y datos en español de LILACS y SCIELO. Se analizaron 53 artículos, más de $50 \%$ de ellos en texto completo, todos los cuales se incluyen por la actualización que ofrecen.

\section{Definición}

El National Health Institute define la CF como un fenómeno de la lactancia o de la infancia, que por lo regular se produce entre los tres meses y los cinco años de edad, relacionada con la fiebre pero sin datos de infección intracraneal o causa identificable. . $^{3-6}$ Cerca de un millón y medio de CF ocurren por año en los Estados Unidos; muchas entre los 6 y 36 meses de edad con un pico a los 18 meses. La incidencia es entre 2 y $5 \%$, y al menos 3 a $4 \%$ de todos los niños en Norteamérica han experimentado al menos una $\mathrm{CF}$ antes de los cinco años.

El riesgo de recurrencia está relacionado con circunstancias como antecedente familiar, infecciones virales tipo influenza A, metapneumovirus, herpes virus tipo 6 y anemia por deficiencia de hierro. ${ }^{7-10}$ Se ha visto que se presenta con mayor incidencia en varones con una relación de 1,5:1. La mortalidad por la propia $\mathrm{CF}$ es nula. El 25 a $40 \%$ de los pacientes con presentan antecedente familiar de crisis febriles. La posibilidad de evolucionar a epilepsia está entre el 2 a 5\%, un poco más alta que la población general. ${ }^{4,5,11-13}$

\section{Clasificación}

Crisis febril simple: representa el $72 \%$ de las crisis febriles y se caracteriza por convulsión generalizada ${ }^{4,14}$, de corta duración (menor de 15 minutos) incluyendo el período posictal, sin compromiso neurológico posterior ni evidencia de infección intracraneana o alteraciones metabólicas severas. Se presenta solo una vez en un período de 24 horas. Pueden tener antecedentes familiares de CF, pero no de epilepsia. ${ }^{11,15}$ Louis y col. plantean elementos clínicos de las crisis febriles simples tales como edad entre seis meses y cinco años, crisis tónico clónica generalizada, cese espontáneo de la crisis en menos de quince minutos, retorno a un estado de alerta luego de la crisis, fiebre $\left(>38^{\circ} \mathrm{C}\right)$, una convulsión en un período de 24 horas y ausencia de alteración neurológica previa. ${ }^{16}$

Crisis febril compleja: comprende el $27 \%$ y se caracteriza por una duración superior a quince minutos, con dos o más episodios en 24 horas, incluido el estado posictal, con antecedentes de alteraciones en el desarrollo psicomotor, historia familiar de epilepsia o presentación de convulsiones. Al examen físico presenta compromiso del sistema nervioso.

Las crisis simples son benignas, mientras que es controversial si las complejas tienen una asociación con el desarrollo de la epilepsia del lóbulo temporal en la vida posterior. Un estudio de cohortes en el Reino Unido encontró que a los 10 años de edad, los niños con crisis febriles no difirieron en el progreso del ámbito académico, el intelecto o el comportamiento en comparación con aquellos sin crisis febriles. ${ }^{17} \mathrm{El}$ 55 a 94\% de los casos son tipo tónicoclónicas, 7 a 33\% tónicas y clónicas de 3 a 28\%. De las convulsiones febriles entre 90 y $93 \%$ son generalizadas, y en menor proporción focales. ${ }^{14,18-25}$

\section{Etiología y fisiopatología}

La crisis febril ocurre en la edad pediátrica, dado que la excitabilidad de la corteza cerebral es alta y depende de la inmadurez de la corteza y la organización córti- 
cosubcortical, y de factores bioquímicos o inmadurez de las diferentes vías de neurotransmisores. Muchos de estos se han descrito en el proceso de CF. Se han documentado dentro de los más frecuentes las alteraciones en la transmisión gabaérgica, dopaminérgica, histaminérgica y serotoninérgica. ${ }^{26}$

La inflamación está emergiendo como un importante mecanismo que contribuye a epileptogénesis. Con respecto a los reactantes inflamatorios, la interleuquina $1 B$ es uno de los principales pirógenos a nivel central. La Il-1ß ha mostrado aumento de la excitabilidad neuronal, actuando en las vías del glutamato y GABA. Sin embargo, no es claro si la IL-1 $\beta$ contribuye al proceso epileptogénico. ${ }^{26}$

En un estudio retrospectivo se comparó la frecuencia de $\mathrm{CF}$ entre quienes recibieron antihistamínico vs el grupo placebo, evidenciándose que la histamina eleva el umbral de ataque y reduce la gravedad y la duración de las crisis. Estos procesos se ven alterados por el uso de antihistamínicos, facilitando la convulsión por fiebre. ${ }^{27}$ Por otro lado el aumento de la temperatura cerebral (hipertermia) podría generar la convulsión por inmadurez de los mecanismos de la termorregulación y una limitada capacidad para incrementar el metabolismo energético celular ante temperaturas elevadas.

Se ha visto que la vacunación es uno de los factores predisponentes en los niños pues se encontró un riesgo cuatro veces mayor de convulsiones febriles después del procedimiento en un período de una a dos semanas. ${ }^{27}$ En cuanto a la vacuna de DPT se han visto entre el primer y tercer día, con la triple viral (MMR) el riesgo aumenta en 1,5 a 3 veces. En un estudio realizado en Dinamarca por Vestergaard y col. se informó que el riesgo relativo (RR) de crisis febriles se incrementa durante las dos semanas posteriores a la vacunación contra la triple viral (2.75; CI 95\%: 2.55-2.97). Este riesgo está aumentado en niños con historia familiar de crisis febril 3.97 veces por 1.000 niños (95\% CI, 2.9-5.4). Quienes presentan convulsión febril posterior a la vacunación contra la triple viral, tienen un riesgo de recurrencia de crisis febriles, mas no de epilepsia. Esto se atribuye al incremento de la replicación viral y la reacción inflamatoria establecida por el huésped. ${ }^{28}$
Las crisis febriles pueden ser una complicación en los niños pequeños que sufren infección con alta carga viral de virus de la influenza H1N1 y se ha postulado que la menor edad puede predisponer a una mayor propensión a secuelas neurológicas graves y a largo plazo el daño neurológico puede ser consecuencia de aumento de citoquinas y alteración de la regulación del sistema inmune debido a la infección. ${ }^{29}$

\section{Factores genétic os}

La CF sigue una herencia autosómica dominante ${ }^{30}$ con un patrón de penetrancia incompleto, por lo que se puede presentar como una herencia multifactorial y poligénica con o sin distribución. Por tanto, su expresión fenotípica puede verse inhibida por otros factores, ya sean ambientales o genéticos.

Los estudios de ligamiento en familias grandes, han permitido identificar cinco diferentes localizaciones, mapeados en los cromosomas: 2, 5, 6, 8 y 19. Tres (FEB1 en el cromosoma 8q13-21, FEB2 en el 19p13, 3 y FEB3 en el 2q23-24) se identificaron en una única gran familia. Otro locus, FEB4, se encontró en el cromosoma 5q14-15 de 47 pequeñas familias y el quinto FEB5, en el cromosoma 6q22-24 se halló en dos familias. Ello indica la heterogeneidad genética de las CF. Si se confrontan los genotipos con los fenotipos clínicos de CF simples y complejas, se observa que los pacientes con genotipo FEB2 cursan preferentemente con CF complejas, sin crisis epilépticas posteriores; mientras que muchos pacientes con genotipo FEB1, FEB2 y FEB4 presentan después crisis epilépticas afebriles, y con genotipo FEB5 solo presentan CF aisladas. $^{22}$

En la Tabla 1 se muestran los hallazgos en biología molecular en las CF y los síndromes epilépticos relacionados que inician con $\mathrm{CF} .{ }^{22}$

La presentación clínica a menudo está dada por episodios de fijación de la mirada, luego supraversión de esta con posterior rigidez generalizada, rara vez focal o con movimientos musculares ${ }^{8-10}$ La duración suele ser breve, entre uno y tres minutos, en el $93 \%$ de los casos. ${ }^{4,14}$ Los principales desencadenantes de 
Tabla I. Genes relacionados con convulsión febril y otros síndromes epilépticos asociados

\begin{tabular}{|c|c|c|c|}
\hline Síndrome epiléptico & Locus & Gen & Proteína \\
\hline Convulsiones febriles, familia I & $8 q 13-q 21$ & & \\
\hline Convulsiones febriles, familia 2 & $19 p \mid 3.3$ & & \\
\hline Convulsiones febriles, familia 3 & $2 q 23-q 24$ & & \\
\hline Convulsiones febriles, familia 4 & $5 q 14-q 15$ & & \\
\hline Convulsiones febriles, familia 5 & $6 q 22-q 24$ & & \\
\hline Epilepsia generalizada con crisis febriles plus, tipo I & $19 q 13.1$ & SCNIB & $\begin{array}{l}\text { Subunidad beta I del canal del } \mathrm{Na}+ \\
\text { dependiente del voltaje (SCNIB) }\end{array}$ \\
\hline Epilepsia generalizada con crisis febriles plus, tipo 2 & $2 q 21-q 33$ & SCN2A & $\begin{array}{l}\text { Subunidad alfa } 2 \text { del canal de } \mathrm{Na}+ \\
\text { dependiente del voltaje }\end{array}$ \\
\hline Epilepsia generalizada con crisis febriles plus, tipo 3 & $5 q 31.1-q 33.1$ & GABRG2 & $\begin{array}{l}\text { Receptor gamma-2 del ácido gamma- } \\
\text { amino butírico }\end{array}$ \\
\hline Epilepsia mioclónica severa del niño & $2 q$ & SCNIA & $\begin{array}{l}\text { Subunidad alfa I del canal del } \mathrm{Na}+ \\
\text { dependiente del voltaje }\end{array}$ \\
\hline Convulsiones febriles y epilepsia lóbulo temporal & Cromosoma 1 y 8 & & \\
\hline
\end{tabular}

una CF son: fiebre, infecciones virales, anemia por deficiencia de hierro, infecciones bacterianas de las vías respiratorias altas, gastroenteritis aguda, exantema súbito, otitis media aguda, infecciones del tracto urinario y reacciones febriles tras la vacunación. ${ }^{7}$ Los diagnósticos diferenciales incluyen las infecciones del sistema nervioso central, factores desencadenantes en niños con epilepsia y otras que se incluyen en la Tabla 2. ${ }^{31}$

\section{Diagnóstic o}

La Academia Americana de Pediatría basada en consensos y publicaciones, no recomienda la realización de exámenes en un niño neurológicamente sano luego de una primera CFS, porque ha demostrado ser ineficaz para predecir ocurrencia de futuras crisis afebriles. ${ }^{32}$

Tabla 2. Diagnósticos diferenciales de

\section{convulsión febril}

\begin{tabular}{|c|c|}
\hline $\begin{array}{l}\text { Infección del sistema } \\
\text { nervioso central }\end{array}$ & $\begin{array}{l}\text { Meningitis } \\
\text { Encefalitis }\end{array}$ \\
\hline \multicolumn{2}{|c|}{$\begin{array}{l}\text { Desencadenantes de la convulsión febril en niños con } \\
\text { epilepsia }\end{array}$} \\
\hline Otras causas & $\begin{array}{l}\text { Enfermedades sistémicas } \\
\text { Bacteremia oculta } \\
\text { Trauma craneoencefálico } \\
\text { Intoxicación } \\
\text { Desequilibrio electrolítico } \\
\text { Hipoglicemia }\end{array}$ \\
\hline
\end{tabular}

Punción lumbar: ante una primera $\mathrm{CF}$ debe considerarse, siempre teniendo presente la edad del niño. Después de los 18 meses no se justifica su práctica rutinaria, mientras que antes de los 12 meses hay que considerarla ya que los signos meníngeos pueden estar ausentes. ${ }^{16}$ Un estudio de cohorte retrospectivo en pacientes de 6 a 18 meses de edad que presentaron una primera CFS entre octubre de 1995 y octubre de 2006, mostró que el riesgo de meningitis bacteriana con esta presentación es muy baja. ${ }^{33}$

Los niños entre seis y doce meses de edad que presentan una convulsión y fiebre, la punción lumbar es una opción cuando no ha recibido las vacunas programadas como se recomienda o el estado de inmunización no se puede determinar a causa de un aumento del riesgo de meningitis bacteriana. La punción lumbar se indica en niños que han recibido tratamiento con antibióticos. ${ }^{32}$

El electroencefalograma puede ser un factor de confusión más que una ayuda diagnóstica. No hay ningún estudio prospectivo que haya podido demostrar la existencia de una relación entre la presencia de unas anomalías paroxísticas en niños que padecen CF y el posterior desarrollo de una epilepsia. ${ }^{16}$

\section{Tratamiento}

El tratamiento inmediato de un niño que ha presentado una $\mathrm{CF}$ debe cumplir un triple objetivo: controlar 
la crisis, tratar el agente causal del proceso febril e informar a los padres.

Para el control de la crisis, existen dos tipos de manejo: a) crítico, en el momento de la convulsión o al poco tiempo de ésta con benzodiacepina (midazolam, diazepam) o ácido valproico y b) profiláctico (terapia continua o intermitente) con ácido valproico, fenitoína y diazepam.

Gran parte de las CFS tienen resolución espontanea (dos a tres minutos) y no requieren manejo adicional. En algunos ocasiones cuando se prolongan ó son recurrentes se procede al manejo farmacológico. ${ }^{34} \mathrm{Si}$ existe gran ansiedad familiar, alto riesgo de recurrencias, crisis prolongadas o difícil acceso al sistema sanitario, se indica profilaxis beneficiándose más los niños con dos o más factores de riesgo.

El fármaco de elección es diazepam oral (DO) o rectal (DR). Los candidatos para tratamiento de rescate se mencionan en la Tabla $3^{30}$, de preferencia con DR al tener una absorción más rápida (alcanza nivel terapéutico a los diez minutos de su administración) y menos efectos secundarios. Debe administrarse al inicio del proceso febril en dosis de $0,33 \mathrm{mg} / \mathrm{k} / \mathrm{dosis}$ (máximo de 7,5 mg/dosis), se puede repetir cada ocho horas si la fiebre persiste durante las primeras 24 horas (el 98\% de las CF ocurren en las primeras 24 horas del comienzo de la fiebre) hasta un máximo de tres dosis. Es dudosa su necesidad en las 24 horas siguientes. ${ }^{34}$

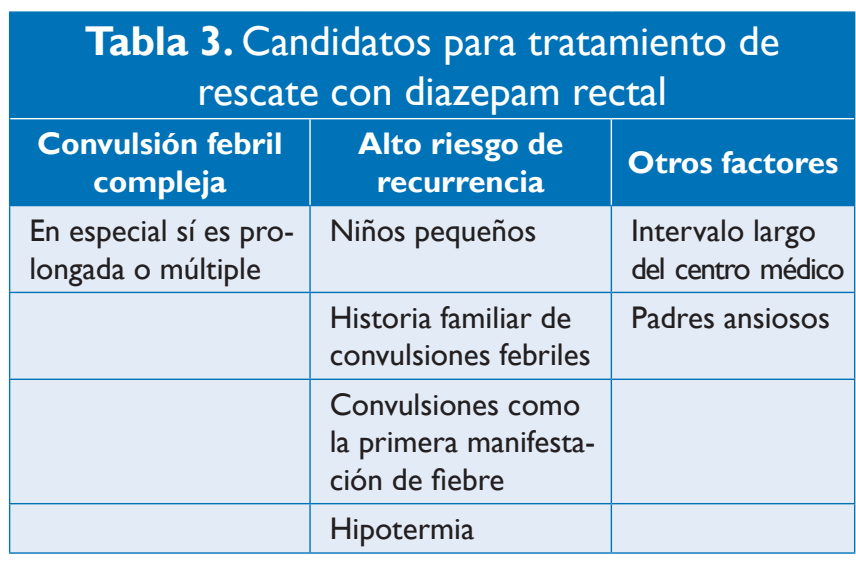

Fuente: Shinnar S, O'Dell C. Febrile seizures. Pediatr Ann 2004 Jun;33(6):394-401.
No hay evidencia de tratamientos para prevenir crisis recurrentes, sin embargo algunos estudios demuestran que la administración continua de fenobarbital y ácido valproico es útil y eficaz, pero se debe evaluar el riesgo/beneficio por las potenciales reacciones adversas a los medicamentos. ${ }^{35} \mathrm{El}$ manejo lógico es disminuir la temperatura pero su efectividad es discutida. Un estudio aleatorio con placebo comparó bajas dosis de diazepam y acetaminofen, sin evidencia que el último prevenga la recurrencia de las crisis. ${ }^{36}$

Beneficios y riesgos del tratamiento con antitérmicos intermitentes: la administración de paracetamol o ibuprofeno en forma pautada (cada cuatro horas) o cuando sube la fiebre, no ha demostrado que reduzca el riesgo de CFS. Aunque son antitérmicos seguros y eficaces, su sobredosificación puede producir importantes efectos adversos. ${ }^{5}$

El manejo general y básico de la reanimación pediátrica es despejar la vía aérea, monitorizar y asegurar un acceso venoso y en caso de ser necesario se administra oxígeno. ${ }^{34} \mathrm{Se}$ inicia diazepam a dosis de $0.5 \mathrm{mg} / \mathrm{k}$ y se puede continuar una infusión máxima de $5 \mathrm{mg} / \mathrm{min}$, el cual se suspende cuando la CF cede. En caso de ser necesario se puede repetir la misma dosis con intervalo de diez minutos.

\section{Medic amentos antic onvulsivantes}

Fenobarbital: es eficaz en la prevención de la recurrencia de las $\mathrm{CFS}^{37}$ y es de elección inicial en las convulsiones neonatales. ${ }^{38}$ Los efectos adversos incluyen hiperactividad, irritabilidad, letargo, alteraciones del sueño y reacciones de hipersensibilidad. Los cambios en el comportamiento pueden ocurrir en $20 \%$ a $40 \%$ de los pacientes y pueden ser tan graves que exigen la interrupción del fármaco. ${ }^{39-43}$

Fenitoína: no ha demostrado ser eficaz en la prevención de la recurrencia de las CFS, incluso cuando se encuentra en el rango terapéutico. ${ }^{44,45}$

Valproatos: el ácido valproico parece tan eficaz en la prevención de las CFS recurrentes como el fenobar- 
bital y bastante más efectivo que el placebo. ${ }^{46-48}$ Los inconvenientes de la terapia incluyen su rara asociación con hepatotoxicidad grave (en especial en niños $<2$ años), trombocitopenia, pérdida o ganancia de peso, trastornos gastrointestinales y pancreatitis. ${ }^{41}$

Diazepam: un estudio controlado doble ciego de pacientes con antecedentes de $\mathrm{CF}$ ha demostrado que la administración de diazepam oral (dada en el momento de la fiebre) podría reducir la recurrencia; se les administro vía oral $(0,33 \mathrm{mg} / \mathrm{k}$, cada ocho horas durante 48 horas) o un placebo en el momento de la fiebre. El riesgo de $\mathrm{CF}$ por persona y año se redujo $44 \%$ con diazepam. ${ }^{48}$

Un estudio más reciente muestra que niños con antecedentes de CF a quienes se les administró diazepam oral en el momento de la fiebre y luego se compararon con un grupo control sin tratar, en los primeros hubo una tasa de recurrencia del $11 \%$ en comparación con $30 \%$ en el grupo control. ${ }^{49}$

Un posible inconveniente de la medicación intermitente es que podría una crisis ocurrir antes de que la fiebre se presente. En ocasiones se puede utilizar la droga profiláctica en los casos de recidivas frecuentes, cuando la ansiedad de los padres es extrema o si existe el riesgo de convulsiones prolongadas o múltiples. ${ }^{50}$ Los efectos adversos de diazepam oral o rectal así como midazolam intranasal y bucal, incluyen letargo, somnolencia y ataxia. La depresión respiratoria es muy rara, incluso cuando se administra por vía rectal. ${ }^{51}$

Antipiréticos: ningún estudio ha demostrado que en ausencia de los anticonvulsivantes reduzcan el riesgo de recurrencia de las CF. Camfield y col. trataron a 79 niños que habían tenido una primera $\mathrm{CF}$ con un placebo, además de instrucciones del uso de antipiréticos (aspirina o acetaminofén) frente a fenobarbital diario más antipiréticos (aspirina o acetaminofén). ${ }^{52} \mathrm{El}$ riesgo de recurrencia fue significativamente menor con fenobarbital, lo que sugiere que el uso de antipiréticos no sirve para prevenir la recurrencia de las CF. La administración de acetaminofén profiláctico durante los episodios febriles es ineficaz para prevenir o reducir la fiebre y prevenir la recurrencia de las $\mathrm{CF} .{ }^{53}$

\section{Conclusiones}

La CF es una patología dentro de un espectro benigno por llamarlo de alguna forma, pero puede llegar a causar angustia tanto en la familia como incluso en el personal médico y paramédico que asiste el evento. Por ello, es de gran importancia tener conocimiento claro del diagnóstico, la clínica y sobre todo del manejo adecuado que debe recibir un paciente con CF en cualquier escenario, basados siempre en las recomendaciones de la American Heart Association (AHA) sobre reanimación pediátrica básica y avanzada.

Se debe recordar siempre que el principal diagnóstico diferencial es meningitis y como tal es pertinente realizar una evaluación clínica y paraclínica exhaustiva y de ser necesario una punción lumbar. Es importante aclarar también que el principal riesgo de la CF es su recurrencia en los porcentajes expuestos con anterioridad.

La Academia Americana de Pediatría recomienda que ninguna terapia anticonvulsivante, continua o intermitente, se recomienda en niños con una o más CF y que debe ofrecerse a los padres un apropiado soporte emocional y educacional para calmar la ansiedad.

\section{Referencias}

1. Izquierdo Bello A, Correa NL, Bolaños CE, Forero FA, Naranjo AR. Protocolo para el estudio y manejo de las crisis febriles PRECOP. CCAP. 2011;10(1):3741.

2. Ochoa-Sangrador C, Gonzalez de Dios J. [Consistency of clinical practice with the scientific evidence in the management of febrile seizures]. Rev Neurol. 2006;43(2):67-73.

3. Jones T, Jacobsen SJ. Childhood febrile seizures: overview and implications. Int J Med Sci. 2007;4(2):110-4

4. Rufo Campos M. Protocolos diagnóstico terapéuticos de la AEP (neurología pediátrica): crisis febriles. [monografía en Internet]. Madrid: AEP; 2008 [citado 9 Jul 2014]. Disponible en: http://www.aeped.es/sites/default/files/documentos/8cfebriles.pdf

5. Steering Committee on Quality Improvement and Management, Subcommittee on Febrile Seizures American Academy of Pediatrics. Febrile seizures: clinical practice guideline for the long-term management of the child with simple febrile seizures. Pediatrics. 2008;121(6):1281-6.

6. Monteny M, Berger MY, van der Wouden JC, Broekman BJ, Koes BW. Triage of febrile children at a GP cooperative: determinants of a consultation. Br J Gen Pract. 2008; 58(549):242-7.

7. Naveed-ur- R, Billoo AG. Association between iron deficiency anemia and febrile seizures. J Coll Physicians Surg Pak. 2005;15(6):338-40.

8. Marcy M, Rogers R, Mackowiak PA. High fevers as a cause of central nervous system sequelae. Pediatr Infect Dis J. 2003;22(3):294-5. 
9. Barone SR, Kaplan MH, Krilov LR. Human herpesvirus-6 infection in children with first febrile seizures. J Pediatr. 1995;127(1):95-7.

10. Chiu SS, Tse CY, Lau YL, Peiris M. Influenza A infection is an important cause of febrile seizures. Pediatrics. 2001;108(4):E63.

11. Casasbuenas OL, Velez A. Guía de manejo para crisis febriles. Rev Méd Sanitas. 2009;12(4):56-8.

12. Guidelines for epidemiologic studies on epilepsy. Commission on Epidemiology and Prognosis, International League Against Epilepsy. Epilepsia. 1993;34(4):592-6

13. Verity CM. Do seizures damage the brain?. The epidemiological evidence. Arch Dis Child. 1998;78(1):78-84.

14. Ripoll Lozano A, Santos Borbujo J. Convulsiones febriles. Protocolo diagnóstico-terapéutico. Bol Pediatr. 2000;40:68-71.

15. American Academy of Pediatrics Steering Committee on Quality Improvement and Management. Classifying recommendations for clinical practice guidelines. Pediatrics. 2004;114(3):874-7.

16. Hampers LC, Spina LA. Evaluation and management of pediatric febrile seizure in the emergency department. Emerg Med Clin North Am. 2011;29(1):83-93.

17. Koyama R, Matsuki N. Novel etiological and therapeutic strategies for neurodiseases: mechanisms and consequences of febrile seizures: lessons from animal models. J Pharmacol Sci. 2010;113(1):14-22.

18. Berg AT, Shinnar S, Darefsky AS, Holford TR, Shapiro ED, Salomon ME, et al Predictors of recurrent febrile seizures. A prospective cohort study. Arch Pediatr Adolesc Med. 1997;151(4):371-8

19. Davis RL, Barlow W. Placing the risk of seizures with pediatric vaccines in a clinical context. Paediatr Drugs. 2003;5(11):717-22.

20. Golnik A. Pneumococcal meningitis presenting with a simple febrile seizure and negative blood-culture result. Pediatrics. 2007;120(2):e428-31.

21. Millar JS. Evaluation and treatment of the child with febrile seizure. Am Fam Physician. 2006;73(10):1761-4

22. Nieto Barrera M, Nieto Jiménez M, Nieto Jiménez E. Convulsión febril. Pediatr Integr. 2007;11(9):753-8.

23. Ishimine P. Fever without source in children 0 to 36 months of age. Pediatr Clin North Am. 2006;53(2):167-94.

24. Hoption Cann SA. Febrile seizures in young children: role of fluid intake and conservation. Med Sci Monit. 2007;13(9):RA159-67.

25. Haslam R. Convulsiones en la infancia. En: Kliegman, Robert M, editor. Nelson tratado de Pediatría. Madrid: Interamericana McGraw-Hill; 1997. p. 2102-4

26. McClelland S, Dube CM, Yang J, Baram TZ. Epileptogenesis after prolonged febrile seizures: mechanisms, biomarkers and therapeutic opportunities. Neurosci Lett. 2011;497(3):155-62.

27. Takano T, Sakaue Y, Sokoda T, Sawai C, Akabori S, Maruo Y, et al. Seizure susceptibility due to antihistamines in febrile seizures. Pediatr Neurol. 2010;42(4):277-9.

28. Vestergaard M, Christensen J. Register-based studies on febrile seizures in Denmark. Brain Dev. 2009;31(5):372-7.

29. O'Leary MF, Chappell JD, Stratton CW, Cronin RM, Taylor MB, Tang YW Complex febrile seizures followed by complete recovery in an infant with hightiter 2009 pandemic influenza A (H1N1) virus infection. J Clin Microbiol. 2010;48(10):3803-5.

30. Shinnar S, O’Dell C. Febrile seizures. Pediatr Ann. 2004;33(6):394-401.

31. Rojas de Recalde L, Montiel de Doldán ME, Sostoa G, Aldana A, Lezcano M. Convulsión febril. Pediatr (Asunción). 2011;38(1):63-7.

32. Subcommittee on Febrile Seizures; American Academy of Pediatrics. Neurodiagnostic evaluation of the child with a simple febrile seizure. Pediatrics. 2011;127(2):389-94.
33. Kimia AA, Capraro AJ, Hummel D, Johnston P, Harper MB. Utility of lumbar puncture for first simple febrile seizure among children 6 to 18 months of age. Pediatrics. 2009;123(1):6-12.

34. Capovilla G, Mastrangelo M, Romeo A, Vigevano F. Recommendations for the management of "febrile seizures": Ad Hoc Task Force of LICE Guidelines Commission. Epilepsia. 2009;50 Suppl 1:2-6.

35. Waruiru C, Appleton R. Febrile seizures: an update. Arch Dis Child. 2004;89(8):751-6.

36. Baumann RJ. Prevention and management of febrile seizures. Paediatr Drugs. 2001;3(8):585-92.

37. Wolf SM, Carr A, Davis DC, Davidson S, Dale EP, Forsythe A, et al. The value of phenobarbital in the child who has had a single febrile seizure: a controlled prospective study. Pediatrics. 1977;59(3):378-85

38. Prasad AN, Seshia SS. Status epilepticus in pediatric practice: neonate to adolescent. Adv Neurol. 2006;97:229-43.

39. Antony JH, Hawke SH. Phenobarbital compared with carbamazepine in prevention of recurrent febrile convulsions. A double-blind study. Am J Dis Child. 1983;137(9):892-5.

40. Knudsen FU, Vestermark S. Prophylactic diazepam or phenobarbitone in febrile convulsions: a prospective, controlled study. Arch Dis Child. 1978;53(8):660-3.

41. Lee K, Melchior JC. Sodium valproate versus phenobarbital in the prophylactic treatment of febrile convulsions in childhood. Eur J Pediatr. 1981;137(2):151-3.

42. Camfield P, Camfield C, Gordon K, Dooley J. What types of epilepsy are preceded by febrile seizures? A population-based study of children. Dev Med Child Neurol. 1994; 36(10):887-92.

43. Bacon CJ, Hierons AM, Mucklow JC, Webb JK, Rawlins MD, Weightman D. Placebo-controlled study of phenobarbitone and phenytoin in the prophylaxis of febrile convulsions. Lancet. 1981;2(8247):600-4.

44. Melchior JC, Buchthal F, Lennox-Buchthal M. The ineffectiveness of diphenylhydantoin in preventing febrile convulsions in the age of greatest risk, under three years. Epilepsia. 1971;12(1):55-62.

45. Wallace SJ, Smith JA. Successful prophylaxis against febrile convulsions with valproic acid or phenobarbitone. Br Med J. 1980;280(6211):353-4.

46. Mamelle N, Mamelle JC, Plasse JC, Revol M, Gilly R. Prevention of recurrent febrile convulsions--a randomized therapeutic assay: sodium valproate, phenobarbital and placebo. Neuropediatrics. 1984;15(1):37-42.

47. Ngwane E, Bower B. Continuous sodium valproate or phenobarbitone in the prevention of 'simple' febrile convulsions. Comparison by a double-blind trial. Arch Dis Child.1980;55(3):171-4.

48. Rosman NP, Colton T, Labazzo J, Gilbert PL, Gardella NB, Kaye EM, et al. A controlled trial of diazepam administered during febrile illnesses to prevent recurrence of febrile seizures. N Engl J Med. 1993;329(2):79-84.

49. Verrotti A, Latini G, di Corcia G, Giannuzzi R, Salladini C, Trotta D, et al. Intermittent oral diazepam prophylaxis in febrile convulsions: its effectiveness for febrile seizure recurrence. Eur J Paediatr Neurol. 2004;8(3):131-4.

50. Offringa M, Moyer VA. Evidence based paediatrics: Evidence based management of seizures associated with fever. Br Med J. 2001;323(7321):1111-4.

51. Pellock JM, Shinnar S. Respiratory adverse events associated with diazepam rectal gel. Neurology. 2005;64(10):1768-70.

52. Camfield CS, Chaplin S, Doyle AB, Shapiro SH, Cummings C, Camfield PR Side effects of phenobarbital in toddlers; behavioral and cognitive aspects. J Pediatr. 1979;95(3):361-5.

53. Chang YC, Guo NW, Huang CC, Wang ST, Tsai JJ. Neurocognitive attention and behavior outcome of school-age children with a history of febrile convulsions: a population study. Epilepsia. 2000;41(4):412-20. 